\title{
A Comparative Teaching of Fractional Calculus and Integer Calculus
}

\author{
Ma Qingxia ${ }^{1 *}$, Lin Xianguang ${ }^{2}$, Li Huijuan ${ }^{1}$ \\ 1 School of Mathematics and Physics, China University of Geosciences, Wuhan 430074, China; \\ 2 College of Biomedical Engineering, South-Central University for Nationalities, Wuhan 430074, China
}

\begin{abstract}
The main purpose of this paper is to give a teaching comparison of fractional calculus and integer calculus. Another purpose of this paper is to help students to understand fractional calculus and integer calculus are highly unified. Starting from the integral order derivative of the elementary power function, the definition of Riemann-Liouville fractional derivative is given. With the theory of integral calculus such as integral mean value theory, Green formula and classical inequality, the fractional differential equations are transformed into integers order differential equation, the differences and relations between two kinds of calculus are expounded. Therefore, it is concluded that fractional derivative and integral derivative can be exchanged under certain conditions.
\end{abstract}

Keywords-Fractional calculus; Integral order calculus; Riemann-Liouville derivative; Riemann-Liouville integral; Comparative teaching

\section{INTRODUCTION}

\section{A. Development Background of Calculus}

Calculus originated in the second half of the seventeenth century. At that time, in order to solve the two practical problems, the tangent problem of the curve and the maximum and minimum problems of the function. Therefore, when calculus was born, it occupied a major position in the entire higher mathematics field. The calculus mainly consists of two parts: differential and integral. The main result is obtained by Newton and Leibniz. The generation of calculus is a great creation in mathematics. It arises from the needs of production technology and theoretical science, which in turn affects the development of production technology and science. Today, calculus is an indispensable tool for scientists and technicians alike. Since the seventeenth century, the concepts and techniques of calculus have been expanded and widely used to solve various practical problems in astronomy and physics, and have made great achievements. Calculus is developed with practical applications. It has been widely used in many branches such as astronomy, mechanics, chemistry, biology, engineering, economics, and other natural sciences, social sciences, and applied sciences. In particular, the invention of computers is more conducive to the continuous development of these applications. Due to the foundation and deeping application of the concept of function, and also due to the needs of the development of science and technology, a new branch of mathematics has been presented after the analytic geometry. And it is named calculus. The position of calculus in the development of mathematics is very important. There is no doubt that it is the greatest creation of all mathematics after European geometry.
Before the generation of calculus, mathematics developed in the period of elementary mathematics. Humans can only study constants, but they can't do anything about them. Only triangles and circles can be discussed geometrically, but nothing can be done for general curves. In the middle of the 17 th century, due to the needs of the development of science and technology, people began to pay attention to the study of variables and general curves. In mechanics, people care about how to determine the instantaneous velocity of a particle based on the path function, or to find the path traveled by the particle based on the instantaneous velocity. Geometrically, one would like to find a way to find the tangent of a general curve and calculate the area of the graph surrounding the general curve. Surprisingly, the problems in different fields boil down to the same model of mathematics: the rate of change of the dependent variable to the independent variable at a certain moment, and the variation of the dependent variable over a certain period of time. The former leads to the concept of differentiation; the latter leads to the concept of integration. Both contain the idea of limits and infinity.

\section{B. Development Background and Application of Fractional Calculus}

Fractional calculus is a branch of calculus that performs fractional differential integration on functions. The so-called fractional calculus does not mean the integral or differential of a fraction or of a fractional function, but means that the order of the differential and the number of integrations are not integers, and it can be any real number. The history of fractional calculus is almost as long as the history of integer calculus. L' Hospital wrote proposed an integer-order derivative to a non-integer case in a letter to Leibnitz September 30, 1695. For example, if $n=\frac{1}{2}$, then $\frac{d^{1 / 2} x}{d x^{1 / 2}}=$ ? It had been 11 years after Leibnitz Published the first paper about calculus, but it was obviously still a difficult question. Leibnitz also could not solve this problem, but he still gave his own opinion: The fractional derivative can introduce some kind of interpolation between the orders of the two integer-order derivatives, and one day it will be a useful result. Since the fractional calculus theory had no exact practical application field, it's development was very slowly over the next hundred years. Although Euler, Bernoulli and other great mathematicians are concerned, fractional calculus is still only some conjecture of pure mathematics. Many mathematicians made a lot of effort on this topic, such as Euler, Lagrange, Fourier, Liouville, Riemman, Weyl. The related concepts and nouns of fractional calculus were gradually exactly defined and 
their characters were discovered, but there were still many imperfections in mathematical theory. Until the modern, fractional calculus had got a great development. And this was due to the development of various applied disciplines, including fluid mechanics, cybernetics, biology, etc. Researchers came to know that fractional calculus theory could solve a various of question in many science fields. In recent years, fractional calculus has been widely used in anomalous diffusion, signal processing and control, fluid mechanics, image processing, soft matter research, seismic analysis, viscoelastic dampers, power fractal networks, fractional sine oscillators, fractal theory, Fractional-order PID controller design. However, since the fractional calculus has historical dependence and global correlation, the numerical computational complexity of the fractional derivative equation is increased.

Fractional calculus theory is much more abstract and profound than classical calculus theory that daily studied by students. During study of fractional calculus, students are easily immersed in boring theoretical proof and lose their interest in learning. Since fractional calculus is an newly developed branch of learning, its application background is rarely introduced in the classic math courses of the university, including Mathematical Analysis, Advanced Mathematics, Differential Equations, etc. In this situation, we must make some changes to let fractional calculus be more acceptable by students. Here we propose a feasible plan to teach fractional calculus. If a teacher is teaching generalized integrals, using the $\Gamma$ function to introduce fractional derivatives and integrals is a better way. He can explain fractional calculus by some specific examples. In these examples, he can show the student that fractional calculus can be transformed into integer calculus under certain conditions. And then the enthusiasm of students will be mobilized to a certain extent, so that students can better grasp fractional calculus and understand the essence of fractional calculus. There are about 1,000 papers on the fractional derivative research each year, and they are growing rapidly. The exchange and academic conferences of fractional calculus theory and application are increasingly frequent.

The fractional derivative has the following advantages: The fractional derivative has a global correlation that can better reflect the historical dependence process of system function development; while the integer derivative has locality, which is not suitable for describing the historical dependence process. The fractional derivative model overcomes the serious shortcomings of the classical integer differential model theory

$$
\frac{d^{\frac{1}{2}}}{d x^{\frac{1}{2}}} 2 \pi^{-\frac{1}{2}} x^{\frac{1}{2}}=2 \pi^{-\frac{1}{2}} \frac{\Gamma\left(1+\frac{1}{2}\right)}{\Gamma\left(\frac{1}{2}-\frac{1}{2}+1\right)} x^{\frac{1}{2}-\frac{1}{2}}=2 \pi^{-\frac{1}{2}} \frac{\Gamma\left(\frac{3}{2}\right)}{\Gamma(1)} x^{0}=\frac{2 \sqrt{\pi} x^{0}}{\sqrt{\pi} 0 !}=1,
$$
\[ \frac{d^{\frac{1}{2}}}{d x^{\frac{1}{2}}} 2 \pi^{-\frac{1}{2}} x^{\frac{1}{2}}=2 \pi^{-\frac{1}{2}} \frac{\Gamma\left(1+\frac{1}{2}\right)}{\Gamma\left(\frac{1}{2}-\frac{1}{2}+1\right)} x^{\frac{1}{2}-\frac{1}{2}}=2 \pi^{-\frac{1}{2}} \frac{\Gamma\left(\frac{3}{2}\right)}{\Gamma(1)} x^{0}=\frac{2 \sqrt{\pi} x^{0}}{\sqrt{\pi} 0 !}=1, \]
which $\quad$ exactly $\quad$ what $\quad$ is $\quad\left(\frac{d^{\frac{1}{2}} d^{\frac{1}{2}}}{d x^{\frac{1}{2}} d x^{\frac{1}{2}}}\right) x=\frac{d}{d x} x=1$. and the experimental results. The use of fewer parameters can achieve good results. When describing complex physics problems, compared with the nonlinear model, the physical meaning of the fractional model is clearer and the expression is more concise.

With regard to the definition of fractional derivatives, many mathematicians start from different angles and give different definitions of fractional derivatives. The rationality and scientificity of its definition have been tested in practice. The development of this branch of mathematics has been widely used in practical problems. So far, there are about four commonly used definitions of fractional derivatives and differentials. These are Riemann-Liouville fractional derivatives, Grunwald-Letnikov fractional derivatives, Caputo fractional derivatives and Miller-Ross Sequention fractional derivatives. In references[2, 5-8], various definitions and properties of fractional calculus were shown. In this article, we used Riemann-Liouville fractional derivative and integral.

\section{AN EXAMPLE OF TRANSFORMING FRACTIONAL CALCULUS INTO INTEGER CALCULUS}

Let $f(x)=x^{k}$, its first derivative is $f^{\prime}(x)=k x^{k-1}$. Any integer order derivative can be obtained by mathematical induction: $\quad \frac{d^{n}}{d x^{n}} x^{k}=\frac{k !}{(k-n) !} x^{k-n}=\frac{\Gamma(k+1)}{\Gamma(k-n+1)} x^{k-n}$. Extending positive integer $n$ to arbitrary real numbers $\alpha$,it is easy to get $\frac{d^{\alpha}}{d x^{\alpha}} x^{k}=\frac{\Gamma(k+1)}{\Gamma(k-\alpha+1)} x^{k-\alpha}$.

For example when $k=1, \quad \alpha=\frac{1}{2}$, semi-derivative of $x$ $\frac{d^{\frac{1}{2}}}{d x^{\frac{1}{2}}} x=\frac{\Gamma(1+1)}{\Gamma\left(1-\frac{1}{2}+1\right)} x^{1-\frac{1}{2}}=\frac{1 !}{\Gamma\left(\frac{3}{2}\right)} x^{\frac{1}{2}}=\frac{2 x^{\frac{1}{2}}}{\sqrt{\pi}}$

Repeating this process, we can get 
III. DISCUSS SOME ANALYTICAL PROPERTIES OF THE SOLUTION, SUCH AS STABILITY, VIBRATION, ETC. OF THE FOLLOWING NONLINEAR FRACTIONAL DIFFERENTIAL EQUATIONS

$$
\left[r(t)\left(D_{0+}^{\alpha} y\right)(t)\right]^{\prime}+p(t)\left(D_{0+}^{\alpha} y\right)(t)+q(t) f\left(\int_{0}^{t}(t-s)^{-\alpha} y(s) d s\right)=0
$$

Equation (2.1) is a nonlinear fractional differential equation with a damping term, which is very difficult to study equation (2.1) directly by using the basic knowledge of fractional calculus. If equation (2.1) can be transformed into ordinary integer differential equations or differential inequalities by means of integer-order calculus, the study becomes simple and straightforward. The following is results of transform:

Lemma2.1[4] : Suppose $y(t)$ is a solution of the equation(2.1), let

$$
F(t)=\int_{0}^{t}(t-s)^{-\alpha} y(s) d s, \alpha \in(0,1), t>0,
$$

then $F^{\prime}(t)=\Gamma(1-\alpha)\left(D_{0+}^{\alpha} y\right)(t)$.

Theorem[4] : Suppose the conditions A1)-A2) are satisfied, let $\omega(t)=\exp \int_{t_{0}}^{t} \frac{r^{\prime}(s)+p(s)}{r(s)} d s$, then the fractional differential equation (2.1)can be transformed into Integer differential inequality

$$
F(t)<F(T)+\Gamma(1-\alpha)\left(D_{0+}^{\alpha} y\right)(T) \omega(T) \int_{T}^{t} \frac{1}{\omega(s)} d s, t \geq T
$$

\section{SUMMARY}

It is very complicated to calculate fractional derivative, so we are trying to find ways to make it easier for students. We hope to establish a four-order formula in calculation of fractional derivatives, which is similar to the calculation of integer-order derivative. We also want to found a chain rule for compound functions to help us in fractional derivative calculation. Fractional derivatives also have properties similar to integer derivatives. For example, fractional derivatives also have linear properties and in accordance with the Leibniz rule. In some certain cases, fractional derivative and integer derivative can be exchanged. And the example is shown in 2.1.

In higher mathematics, many functions can be expanded into triangular series or power series. If certain conditions are met, they can be derived item by item, and the convergence of the series after the derivation is able to be judged. We can also use similar methods to deal with fractional calculus of function, which also can be expanded to series and is still not out of the scope of classical analysis.

Generally speaking, it is difficult to study fractional differential equations by using fractional calculus theory. But if you use the integer calculus theory, you can transform fractional differential equations into normal differential equations in the study. The fractional-order operator unifies the differentiation and integration in form, which is not available in classical calculus.

The main idea of fractional calculus is to generalize the classic integer calculus, extending the concept of calculus to the entire real axis, or even the entire complex plane. But because the various manner of extending, people give different definitions of fractional calculus according to different needs. Till now, no unified definition form for fractional calculus was widely accepted, which has caused certain difficulties in the research and application of fractional calculus.
The establishment of calculus has greatly promoted the development of mathematics. Many problems that could not be solved with elementary mathematics In the past were settled by calculus. This showed the overwhelming power of calculus. Human will never stop the discover of nature, and the related science will also progress. The discipline of calculus has also been developing in modern times, and the level of human understanding of calculus is constantly deepening day by day. Fractional calculus mainly includes fractional differential and fractional integrals in the narrow sense, and it contains both fractional difference and fractional order and quotient in a broad sense. In recent years, the theory of fractional calculus has been successfully applied to various fields. It is gradually found that fractional calculus can describe some non-classical phenomena in the natural science and engineering applications. In the teaching of calculus, appropriately introducing the basic content of the fractional calculus in the current mathematics subject in the classroom is very useful. In this way, we can expand the teaching content of the university mathematics curriculum, which is conducive to cultivating students' interest and enthusiasm in learning mathematics. And this teaching method can let students realize that the integration of integrals and derivatives from integer order to fractional order will further expand the application range of the derivative. During this progress, we will have a deeper understanding of many complex phenomena in nature.

\section{ACKNOWLEDGEMENTS}

This work was supported by National Natural Science Foundation 11701533 and Open Fund of Hubei Key Laboratory of Medical Information Analysis and Tumor Diagnosis \& Treatment PJS140011710. 


\section{REFERENCES}

[1] Wu Guangqiang, Huang Huanjun, Ye Guanghu. Semi-active control of automotive air suspension based on fractional calculus [J]. Journal of Agricultural Machinery, 2014, 7: 19-25. (In Chinese)

[2] Kilbas A A, Srivastava H M, Trujillo J J. Theory and application of fractional differential equations[M]. Amsterdam: Elsevier, 2006.

[3] Lv Xin, Liu Guangting. Comparison of several common fractional calculus definitions [J]. Journal of Inner Mongolia Normal University, 2017, 46(4): 479-482. (In Chinese)

[4] Ma Qingxia, Liu Anping. Oscillation criteria of nonlinear fractional differential equations with damping term[J]. Mathematica Applicata, 2016, 29(2): 291-297.

[5] Podlubny I. Fractional differential equations [M]. San Diego: Academic Press, 1999.

[6] Samko S G, Kilbas A A , Marichev D I. Fractional intergrals and derivatives: theory and applications [M]. USA: Gordon and Breach Science Publishers, 1993.

[7] Millers K S, Ross B. An introduction to the fractional calculus and fractional differential equations[M]. New York: John Wiley, 1993.

[8] Oldham K B, Spanier J. The fractional calculus [M]. Academic Press, New York, 1999.

[9] Zheng Zuxiu. Development and Application of Fractional Differential Equations[J]. Journal of Xuzhou Normal University (Natural Science Edition),2008,26:1-10. (In Chinese) 